https://doi.org/10.22319/rmcp.v9i3.4329

Artículo

\title{
Valorización de una Indicación Geográfica Protegida. El caso de la carne de la Sierra de Guadarrama, España
}

\section{Valuation of a Protected Geographical Indication. The case of the meat of the Sierra de Guadarrama, Spain}

Mario del roble Pensado-Leglise ${ }^{\mathrm{a} *}$

Javier Sanz-Cañada ${ }^{b}$

a Instituto Politécnico Nacional, IPN, Ciudad de México, México.

${ }^{\mathrm{b}}$ Centro Superior de Investigaciones Científicas, CSIC, Madrid, España.

* Autor de correspondencia: mpensado@ipn.mx

\section{- Resumen:}

En este trabajo se analiza la relación entre la calidad y el valor de un bien de indicación geográfica protegida (IGP) con respecto a su precio, tomando la experiencia de veinte años de los productores de carne de la Sierra de Guadarrama. Mediante una investigación cualitativa a través de la técnica de enfoque grupal "focus group" y del uso del software Atlas.ti, se encontró que la carne de la Sierra de Guadarrama cuenta con varios factores que fortalecen la confianza del consumidor de la Comunidad de Madrid al bien de especialidad, como son: la identidad cultural, el apego por ser un producto local, buenas prácticas de sanidad animal y de inocuidad de la carne. Pese al prestigio social del bien de especialidad, existen limitaciones para alcanzar mejores precios pagados a los ganaderos, debido a una falla de mercado basada en la falta de información completa al consumidor (sobre la importancia de la ganadería para la conservación del patrimonio paisajístico ambiental); así como existir una ventana de oportunidad para mejorar la organización de la asociación y mayor acción colectiva de los productores que redunde en mejorar su forma de gobernanza, en particular su capacidad de negociación con los demás actores de la fase de 
comercialización, y con ello pueda haber un mejor equilibrio entre la calidad, valor y precio para el ganadero participante en la IGP.

- Palabras clave: Indicaciones Geográficas Protegidas, Bienes de especialidad, Calidad, Precios, Denominación de origen, Fallas de mercado.

\begin{abstract}
- Abstract:
This paper analyzes the relationship between the quality and value of a well-Protected Geographical Indications (PGI) with respect to its price, taking the experience of $20 \mathrm{yr}$ of beef producers of the Sierra de Guadarrama. Through a qualitative research through the focus group technique and the use of the software Atlas.ti, it was found that the meat of the Sierra de Guadarrama has several factors that strengthen consumer confidence in the Autonomous Community of Madrid to the good and specialty, such as: cultural identity; the attachment to be a local product; good practices of animal health and meat safety. Despite the social prestige of the specialty, there are limitations to achieve better prices paid to farmers due to a market failure based on the lack of complete information to the consumer (importance of livestock to the preservation of the heritage landscape assessment), as well as a window of opportunity to improve the organization of the association, greater collective action of producers which helps to improve the form of governance, in particular their capacity to negotiate with other actors in the marketing phase and this may be a better balance between the quality, value and price to the farmer of the PGI.
\end{abstract}

Key words: Protected Geographical Indications, Goods specialty, Quality, Prices, Protected designation of origin, Market failures.

Recibido 17/11/2016.

Aceptado 09/11/2017.

\title{
N Introducción
}

La calidad de un bien se reconoce por medio de una convención o de un acto social entre concurrentes que acuerdan, aceptan la calidad y reconocen el valor de un bien que se traduce a un precio. Salais y Thevenot $^{(1)}$ y otros ${ }^{(2,3)}$ estudiaron la relación entre la calidad de un bien 
alimentario según el tipo de convenciones sociales que la rigen. En un bien con Indicación Geográfica Protegida (IGP), el valor corresponde a la calidad ${ }^{(4)}$, tipicidad ${ }^{(5)}$ y a las convenciones $^{(6)}$ inherentes a un proceso histórico de construcción social de la calidad de un producto con sello de exclusividad a un lugar geográfico ${ }^{(7)}$.

IGP, es "el nombre de un lugar determinado, que sirve para designar un producto agrícola o alimenticio originario de dicha región, lugar o país, que posea una cualidad determinada, una reputación u otra característica que pueda atribuirse a dicho origen geográfico, y cuya producción, transformación o elaboración se realice en la zona geográfica" ${ }^{\prime(8,9)}$. Para ser un bien IGP basta que alguna de las fases de la producción esté vinculada al área geográfica ${ }^{(10,11)}$.

En la globalización, los cambios en el patrón de consumo alimentario expandieron la demanda de bienes de especialidad ${ }^{(12)}$, y se crearon nuevos nichos de mercado para productos locales en el mercado global ${ }^{(13-16)}$. La nueva demanda de bienes IGP, con reputación social de calidad territorial ${ }^{(17)}$ multiplicó las marcas colectivas: en el comercio justo, de bienes "slow food"(18); bienes de circuitos cortos; de bienes con buenas prácticas ambientales o de agricultura orgánica ${ }^{(19)}$; ligados a la acción colectiva y a capital relacional basado en la proximidad ${ }^{(20)}$. Su dinamismo hizo incluirlos en las negociaciones comerciales multilaterales desde los 80 s a la fecha ${ }^{(21)}$.

El bien IGP distingue a un sistema agroalimentario local (SIAL) ${ }^{(22)}$ pero no garantiza mejor precio al productor. Requiere de una estrategia eficaz de acción colectiva local que facilite, gestione, coordine e innove con nuevos arreglos institucionales entre productores y consumidores. En un bien IGP, la renta absoluta para el productor es parte del precio productivo que incluye el pago por el usufructo de los derechos de exclusividad ${ }^{(23,24)}$ y es similar a la naturaleza de un bien-club (bien con precio especial, de un grupo de productores con certificado de usufructo de sus derechos exclusivos). Si hay falla de mercado o si el grupo que reclama su renta absoluta posee insuficiencia organizativa, no hay capacidad de mejorar los precios a productores. En España, los bienes IGP/DOP crecieron de 12 en 1987 a 168 en $2012^{(25)}$ debido a los incentivos de los apoyos al Desarrollo Rural de la Política Agrícola Común (PAC) otorgados desde 1992, pero no todos mejoraron los precios a productores de manera sustantiva.

De la carne vacuna española, un estudio de $2017^{(26)}$ indicó que el $89 \%$ de consumidores españoles la aprecia por su sabor, ser fuente de proteínas y con valor nutricio; el $52.4 \%$ del total considera la carne de vacuno española como la mejor (por la crianza, la alimentación, la calidad de la carne y su inocuidad). El $90 \%$ de los españoles consideró al origen y a la alimentación como principales factores para que la carne sea jugosa. El $48 \%$ aprecian mucho se identifique el origen de la carne. El $60 \%$ de españoles la consumen al menos una vez a la semana; el $63 \%$ está conforme con la cantidad consumida y el $73 \%$ está conforme con la relación calidad-precio. En los factores que influyen en la compra, el precio está en cuarto lugar y en cambio el $51 \%$ lo hace por su propio gusto. La carnicería de barrio y el 
supermercado son predilectos para compra de carne de vacuno y al $90 \%$ le gusta comprar en fresco al mostrador.

La carne de la Sierra de Guadarrama significó alrededor de $2.3 \%$ de las unidades de explotación ganadera bovina españolas de carne IGP; el $4.5 \%$ en volumen producido y el $5 \%$ en valor de ventas con respecto al total de bienes cárnicos IGP nacional ${ }^{(27)}$. La IGP inició en 1994, por el interés de los ganaderos aprovechando la oportunidad de un programa de fomento a dar una marca de calidad a su carne por la Comunidad de Madrid. Luego, se logró el reconocimiento oficial de la Unión Europea y para 2016 había 368 unidades de explotación. Dentro de los productores hay 239 que tienen cebaderos y vacas nodrizas en la sierra y 129 solo incluyen el libre pastoreo y las vacas nodrizas. No obstante, los ganaderos de tiempo completo son menos de un centenar ${ }^{(28)}$. En 2016, eran cuatro los mataderos supervisados por la IGP y ocho salas de despiece; había 146 puntos de venta registrada por la IGP de los cuales el $52 \%$ del total eran carnicerías y tiendas locales, y el resto eran sucursales de supermercados, tiendas de autoservicio e hipermercados. El total de animales sacrificados fue 5,176 cabezas (1,566.7 t de carne); es decir, en promedio $302.70 \mathrm{~kg}$ de carne en canal producida en 15 meses. Otros actores de la IGP son el entradero (comisionista), el matadero (maquila independiente) y la sala de despiece de distribuidora o autoservicio. El consumo no es exclusivo de Madrid (Ciudad), sino también la venta se hace en localidades de la Sierra, donde hay carnicerías, tiendas y restaurantes. El precio de la carne de IGP pagado al ganadero es mayor al $2 \%$ con respecto al de la carne vendida en la lonja regional (bolsa comercial de productos cárnicos en físico), lo cual es poco para el ganadero, dado el mayor tiempo dedicado y el pago del costo adicional de la certificación IGP. Además, no se reconoce el valor del servicio ambiental de la práctica ganadera serrana, que contribuye a conservar en buen estado el pastizal y disminuir los riesgos de incendios forestales. Esto es porque al ser la zona de la IGP de la Comunidad de Madrid: una parte corresponde al Parque Nacional Sierra de Guadarrama (ubicada en la Comunidad de Madrid), que posee gran riqueza de paisaje y patrimonio histórico cultural ${ }^{(29)}$, y la otra es una zona del occidente de la Comunidad de ganadería extensiva.

Este trabajo tiene por objeto analizar los aspectos de valorización que limitan un mejor precio a los ganaderos de la IGP de la carne de la sierra de Guadarrama.

\section{Material y métodos}

La métodología fue de carácter cualitativo, y consistió en entrevistas guiadas o dirigidas por medio de un cuestionario y la realización de un taller de enfoque grupal. La actividad está 
concentrada en poco menos de 20 grandes productores que participan en forma decisiva en la asociación de la IGP, por lo cual, se omiten nombres de personas y lugares por ser información reservada. En primer lugar, se efectuaron diecinueve entrevistas semidirigidas a los diversos actores participantes de la cadena de valor y se completaron alrededor de catorce horas de grabación. Ocho de cada diez entrevistados eran mayores de 55 años, por lo que se observa la necesidad de un cambio generacional en aquellos que intervienen en la cadena de valor. Por grupos de actividad, se entrevistaron a productores pequeños, productores grandes, "entraderos" (tipo de comisionista acopiador), personal técnico y administrativo de carnicerías independientes y tiendas de autoservicio, así como también a personal técnico que labora para la IGP y funcionarios responsables de la Comunidad de Madrid. Luego, se procedió a la transcripción de las entrevistas se editaron y se sistematizó la información y se analizó con la técnica de análisis heurístico mediante el manejo del Atlas.ti., versión 7.5.15. ${ }^{(30)}$. En segundo lugar, se utilizó la técnica de enfoque grupal ${ }^{(31)}$ con una pequeña muestra representativa de ganaderos. La sesión se realizó con cinco actores: dos grandes productores (participantes en las dos cooperativas que existen), un pequeño productor, el presidente de la Asociación de ganaderos de la IGP y el Gerente de la IGP. La importancia de los actores participantes en el enfoque grupal, es que ellos tenían injerencia en el manejo de las unidades de producción asociadas, que significaba alrededor de la mitad del valor de ventas anual de la IGP o eran protagonistas institucionales y sociales.

\section{Resultados}

\section{- Análisis con Atlas.ti •}

A partir de la información recabada con las entrevistas que arrojaron frases con ideas claves y de acuerdo a su sitematización con la Figura 1 (obtenida del análisis con Atlas.ti), se desprendió la existencia de cinco elementos determinantes en la trayectoria de IGP de la carne de la Sierra de Guadarrama, a saber: 1) identidad cultural con la Comunidad de Madrid por ser un bien de especialidad típico; 2) calidad sanitaria (certificación de calidad que requiere altos estándares en todas las fases de la cadena de valor); 3) subvenciones de la PAC (inversión en equipo e infraestructura y promoción comercial), mejorando la cadena de valor 
y el reconocimiento social en la comunidad de Madrid; 4) manejo productivo que combina razas; 5) prácticas productivas semi-intensivas; 6) prácticas sociales productivas amables con el ambiente, contribuyendo a la disminución de riesgos de incendios.

Figura 1: Dinámica de la IGP de la carne de la Sierra de Guadarrama en Altas.ti

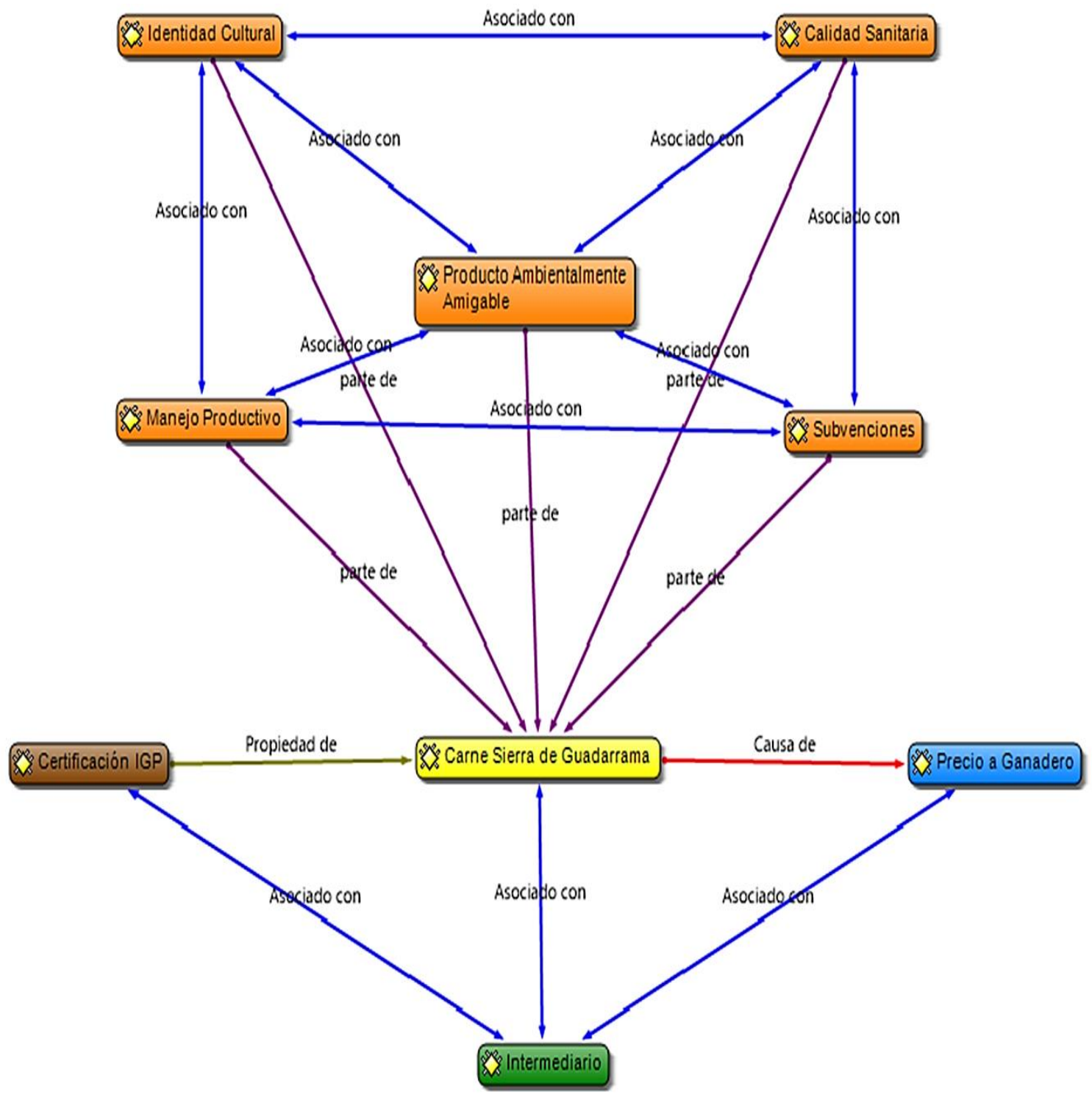

Fuente: elaboración de MRPL y JABD en software Atlas ti

El reconocimiento de estos elementos, como puntos principales en la construcción social de calidad de la IGP de la carne de la Sierra de Guadarrama se valorizan y forman parte del precio (la intermediación comercial está relacionada tanto con la certificación de la IGP, 
como con la calidad de la carne). Así, el importe se basa tanto en el precio de carne de vacuno regional, como en las negociaciones relacionadas con el sobreprecio pagado al ganadero de la IGP. En la zona, se pueden encontrar algunos productores que producen de manera simultánea, tanto ganado vacuno sin registro como con registro de IGP, así la intermediación comercial es el nudo crítico de la cadena que puede limitar o impulsar (Figura 1).

\section{- Resultados con el método de enfoque grupal •}

El taller de enfoque grupal se realizó con cinco participantes; se presentó un diagnóstico general de la cadena y se hicieron preguntas para establecer dos momentos en la discusión; en la primera ronda de discusión, hubo consenso al identificar como los principales problemas para mejorar la rentabilidad de los productores, tanto los altos costos productivos como la debilidad de la asociación de productores en la negociación de precios con el mercado, básicamente por el predominio de la venta individual a intermediarios. También se señaló la necesidad de mejorar la eficacia y cantidad de los apoyos oficiales, y deficiencias en el manejo de los animales (Cuadro 1). Entre las principales soluciones se mencionaron mejorar el funcionamiento de la asociación, integrar verticalmente la cadena (desde la producción hasta la carnicería), mejorar el circuito de comercialización incluyendo ventas directas o por internet, mayor apoyo gubernamental para la promoción comercial, fomentar el circuito de proximidad en la comercialización, controlar la fauna salvaje, mejorar la regulación del uso turístico urbano de la Sierra, y establecer un pago por conservación del medio ambiente. 


\section{Cuadro 1: Resultados de rondas de participación del enfoque grupal}

\begin{tabular}{|c|c|c|c|c|c|}
\hline \multicolumn{6}{|c|}{ I Cómo jerarquizas problemas actuales de la asoc de productores y Cuáles consideras que son sus soluciones? } \\
\hline \multirow[b]{2}{*}{ Problemas } & A & B & C & D & E \\
\hline & & & & & \\
\hline 1 & Cebo en Común de Animales & Comercialización & Fallas Comercialización & $\begin{array}{l}\text { Los terneros salen a la } \\
\text { Sierra }\end{array}$ & Cuesta mucho cebar \\
\hline \multirow{2}{*}{2} & Comercialización conjunta & Cebo para animales & Falta Publicidad & $\begin{array}{l}\text { Pastos de la Comunidad } \\
\text { son muy caros }\end{array}$ & Cuesta vender \\
\hline & $\begin{array}{l}\text { Falta apoyo oficial de la } \\
\text { Comunidad de Madrid }\end{array}$ & $\begin{array}{l}\text { Mal manejo de animales al } \\
\text { destete }\end{array}$ & Duplicación de socios & Pocos cebadores & $\begin{array}{l}\text { Deberían apoyar más a los } \\
\text { cebaderos }\end{array}$ \\
\hline \multirow{2}{*}{4} & Excesiva Fauna Salvaje & Problemas sanitarios & $\begin{array}{l}\text { Evitar intermediarios en la venta } \\
\text { de producto }\end{array}$ & Piensos Caros & es dificil cebar \\
\hline & $\begin{array}{l}\text { Excesiva burocracia apra el } \\
\text { ganadero }\end{array}$ & ND & $\begin{array}{l}\text { Incluir una vía de venta en internet } \\
\text { dentro de la venta general }\end{array}$ & $\begin{array}{l}\text { Problemas } \\
\text { medioambientales }\end{array}$ & Está dficil para los jóvenes \\
\hline 5 & $\begin{array}{l}\text { NO tener que pagar gastos de } \\
\text { comunidad }\end{array}$ & ND & ND & Comercialización irregular & Unirse para cebo en común \\
\hline \multicolumn{6}{|l|}{ Soluciones } \\
\hline 1 & Cebadero común & Comercialización conjunta & Comercialización conjunta & Pastos gratis & $\begin{array}{l}\text { Unión para compras } \\
\text { comunes }\end{array}$ \\
\hline 2 & Comercialización conjunta & $\begin{array}{l}\text { Compras en común para unificar } \\
\text { la alimentación }\end{array}$ & Mas promoción publicitaria & $\begin{array}{l}\text { Mejor Manejo Animal en la } \\
\text { Sierra }\end{array}$ & $\begin{array}{l}\text { Unión para } \\
\text { comercialización conjunta }\end{array}$ \\
\hline 3 & $\begin{array}{l}\text { Mas recursos oficiales de la } \\
\text { Comunidad de Madrid }\end{array}$ & ND & $\begin{array}{l}\text { Mejor situación económica del } \\
\text { páis incrementa consumo }\end{array}$ & ND & $\begin{array}{l}\text { Por falta de compras } \\
\text { comunes }\end{array}$ \\
\hline 4 & Control de Fauna Salvaje & Control Fauna Salvaje & Venta de proximidad y directa & ND & apoyar a la gente joven \\
\hline 5 & Reducir papeleo a gandaderos & ND & ND & ND & ND \\
\hline 6 & $\begin{array}{l}\text { Eliminar los pliegos a pastos de } \\
\text { la comunidad de Madrid }\end{array}$ & ND & ND & ND & ND \\
\hline \multicolumn{6}{|c|}{ II Cómo mejorar la asociatividad para beneficio y sostenibilidad de la IGP? } \\
\hline \multirow[t]{2}{*}{1} & Cebadero Común & $\begin{array}{l}\text { Constituir una asociación que } \\
\text { unifique la alimentación y la } \\
\text { compras conjuntas }\end{array}$ & $\begin{array}{l}\text { La asociación se mejoraría con } \\
\text { mayor implicación de los socios y } \\
\text { esto se puede conseguir con } \\
\text { mejoría económica que conlleve } \\
\text { mejora de la comercialización }\end{array}$ & $\begin{array}{l}\text { Mejorar la } \\
\text { comercialización para } \\
\text { ganar ingresos }\end{array}$ & $\begin{array}{l}\text { Asociación para } \\
\text { comercialización }\end{array}$ \\
\hline & Comercialización conjunta & $\begin{array}{l}\text { Constituir una comercializadora } \\
\text { que maneje todo el ganado de } \\
\text { la IGP }\end{array}$ & $\begin{array}{l}\text { La IGP tendría que incluir entre sus } \\
\text { socios a los comercializadores } \\
\text { (carnicerías y supermercados, etc) } \\
\text { asíse conseguiría fidelidad de los } \\
\text { puntos de venta }\end{array}$ & $\begin{array}{l}\text { Compra en común que } \\
\text { reduzca precios de piensos }\end{array}$ & $\begin{array}{l}\text { Considerar a los jóvenes } \\
\text { en todos los programas }\end{array}$ \\
\hline 3 & $\begin{array}{l}\text { Mas presupuesto para } \\
\text { promoción de marca de la IGP }\end{array}$ & Reforzar promoción de la Marca & ND & ND & ND \\
\hline 4 & $\begin{array}{l}\text { Reducir papeleo para el } \\
\text { ganadero }\end{array}$ & $\begin{array}{l}\text { Ayudas de la administración, en } \\
\text { cuanto el fondo de extensión es } \\
\text { realmente el principal } \\
\text { responsable de la cadena }\end{array}$ & ND & ND & ND \\
\hline 5 & $\begin{array}{l}\text { Necesidad de solo una dirección } \\
\text { General de Ganadería }\end{array}$ & Medio ambiental & ND & ND & ND \\
\hline 6 & ND & ND & ND & ND & ND \\
\hline
\end{tabular}

Fuente: elaboración propia con base en resultado de Taller Focus Group, mayo, 2015 
En la segunda ronda de discusión (cómo mejorar la asociación), dos fueron los temas centrales: tener un proyecto industrial de cebadero común y que la asociación pueda funcionar con mayor dinamismo (compra de insumos y venta asociativa), para posicionarse mejor en el mercado. Otras propuestas fueron incluir a otros actores como son los comercializadores, mejorar los apoyos a jóvenes productores, aumentar recursos para la conservación medio ambiental, mayor promoción oficial de la marca, así como impulsar una simplificación administrativa.

\section{| Discusión |}

La etiqueta de calidad y origen que ofrece la IGP, son los principales atributos de la certificación $^{(32)}$. En diversos estudios europeos se indica que las nuevas familias jóvenes de menor tamaño y mayor educación tienen preferencia por las carnes de $\operatorname{IGP}^{(33)}$. Si bien la valorización es importante, no hay que perder de vista que la certificación implica un costo por lo que el margen de ganancia es mínimo. De acuerdo con Gracia y Pérez ${ }^{(34)}$ dichos atributos generaban elasticidades bajas; en 2002 encontraron que un aumento de $1 \%$ por concepto del origen geográfico, en la importancia asignada por tener una Denominación de Origen Protegida (DOP) se traducía a pagar $0.105 \%$ más por la carne de ternera, de suerte que los consumidores que adquirían carne de ternera con DOP pagaban solo un $2.6 \%$ más que el precio medio de la carne de ternera. En otro estudio de carne de oveja (marca Ternasco de Aragón), se observa la competencia desleal entre la carne producida con registro de IGP y aquélla en la que se publicita su procedencia pero no tiene certificación ${ }^{(35)}$. En este caso, la falla de mercado radica en una información incompleta al consumidor ${ }^{(36)}$, lo que es posible también sea el caso para la carne de la Sierra de Guadarrama; al respecto, la importancia del capital social y de la acción colectiva en la valorización es fundamental para la distinción de la calidad y su aporte a los bienes públicos territoriales.

Por otra parte, el capital social en la valorización es fundamental para la distinción de la calidad y su aporte a los bienes públicos territoriales. Barjolle y Sylvander ${ }^{(37)}$ indican que la coordinación en la gestión del mercado es la relación entre la acción colectiva y el ámbito de cada empresa para variar la calidad del producto y adaptarla a su propia estrategia, ya que esto permite a las empresas manejar la competencia en los mercados segmentados. Por eso, la coordinación efectiva de los ganaderos de la Sierra de Guadarrama puede convertirse en una ventaja competitiva y dota de mayor tipicidad social al producto IGP. Belletti et al ${ }^{(38)}$ plantean que es útil la cohesión organizativa de los productores de la IGP porque permite 
mostrar la calidad de su producto, capitalizando su reputación social de calidad como recurso intangible colectivo frente a los consumidores. En este caso, el funcionamiento del producto IGP es como bien club $^{(39)}$ pero dentro de un ambiente de heterogeneidad económica productiva, lo cual es otra condicionante en las posibilidades de éxito o fracaso de cohesionar al esfuerzo organizativo y de coordinación de los ganaderos.

La valoración del IGP genera o fortalece bienes públicos territoriales, pues como sello de calidad colectiva se distingue por una tipicidad, pero también representa un recurso intangible territorial que patrimonializa el paisaje natural y sociocultural. Linck, et $a l^{40)}$ plantean que la valoración del bien público generado por la IGP se basa en ofrecerles las dimensiones patrimoniales del territorio visto como un recurso complejo - que asocia recursos producidos y ambientales con conocimientos técnicos y relacionales específicos - y compartido en su ámbito espacial y social. Desde la óptica de las políticas públicas, se podría proponer subsidios específicos para las prácticas diferenciales de manejo del ganado que pasta en las zonas incluidas en el Parque Nacional de Guadarrama y su área de influencia.

Belleti, et $a l^{(41)}$ indican que la valoración del bien IGP como impulsor de la patrimonialización del bien público territorial, juega un papel importante en activar y apoyar procesos de desarrollo sostenible en áreas rurales, pero tiene sus riesgos, y los resultados están en función de diferentes áreas de intervención para la acción colectiva y su relación con las políticas públicas de un producto IGP como bien con naturaleza multifuncional ${ }^{(42)}$.

Los resultados obtenidos a partir de un análisis empírico, coinciden con otros trabajos científicos de indicaciones geográficas españolas y europeas, que señalan al déficit de innovación social en la gobernanza territorial como un factor adicional que impide generar una suficiente renta a pesar del esfuerzo en la construcción de instituciones colectivas en sistemas agroalimentarios locales ${ }^{(43)}$.

\section{Conclusiones e implicaciones}

Después de 20 años la IGP de la carne de la Sierra de Guadarrama posee reputación social por ser un bien saludable, con calidad local e identidad cultural territorial. Sin embargo, este aprecio social no se traduce en mejor precio para los ganaderos, y ello pone en riesgo a la actividad a largo plazo. Un aspecto del problema es la falla de mercado, porque no se reconoce a los servicios ambientales de la ganadería serrana en conservar la biodiversidad y el valor del patrimonio cultural de la Sierra de Guadarrama. Otro aspecto son las fallas 
organizativas, lo que puede ser superado con asociatividad en la comercialización e innovación social en las relaciones entre productores y consumidores; ello implica fortalecer el capital relacional, mejorar la coordinación y elevar su capacidad de negociación en el mercado. Resolver ambos aspectos es esencial para dar sostenibilidad a la actividad en armonía con buenas prácticas ambientales e inocuidad alimentaria, que ofrezca un bien de especialidad para el consumidor de la Comunidad de Madrid.

\section{Agradecimientos}

A CONACYT por la estancia de año sabático del Dr. Mario del Roble Pensado-Leglise en el IEGD-CCHS del CSIC, Madrid, España, en el marco del Proyecto SIP 20151928 del Instituto Politécnico Nacional, CIIEMAD, México. La redacción final se hizo con el Proyecto SIP 20181987. Se agradece al M. en C. Juan Antonio Bautista Dávila por su labor técnica en el ejercicio de Atlas.ti.

\section{- Literatura citada}

1. Salais R, Thevenot L éds. Le travail. Marchés, règles, conventions. Paris, INSEEEconomica; 1986.

2. Eymard-Duvernay F. La qualification des produits. In: Salais R, Thévenot L éds. Le travail: marchés, règles, conventions. Paris, Insee-Economica; 1986:239-247.

3. llaire G. Emergence d'un nouveau systeme productif en agriculture. Rev Canad Econ Rur 1996;44(4):461-491. https://www.researchgate.net/publication/229915970_ Emergence_d'un_nouveau_systeme_productif_en_agriculture. Accesed Jun 14, 2015.

4. Sylvander B, Lagrange L, Monticelli C. Les signes officiels de qualité et d'origine européens. Quelle insertion dans une économie globalisée? Rev Economie Rurale 2007;(299):7-23. 
5. Sanz-Cañada J. Calidad y signos distintivos. Las denominaciones de origen de aceite de oliva en España. En: Sanz CJ editor. El futuro del mundo rural. Madrid: Ed. Síntesis; 2007:175-198.

6. Allaire G. L' économie de la qualité, en ses territoires, ses secteurs et ses mythes, Géogr Econ Soc 2002;4(2):155-180.

7. Francoise J. La construction sociale de la qualité alimentaire dans un lien producteur consommateur le cas du marché public de Rimouski au Québec. [Thesis maître ès arts] Quebec: Université du Québec À Rimouski; 2011.

8. Comunidad Económica Europea. CEE. Reglamento CEE 510/2006, 20 de marzo de 2006.

9. Sidali KL, Hemmerling S. Developing an authenticity model of traditional food specialties: Does the self-concept of consumers matter? Brit Food J 2014;16(11):16921709. http://dx.doi.org/10.1108/BFJ-02-2014-0056. Accesed Jun 14, 2015.

10. Kvam GT, Magnus, T, Stræte EP. Product strategies for growth in niche food firms. Br Food J 2014;116(4):723-732.

11. Lin L, MaoPC. Food for memories and culture - A content analysis study of food specialties and souvenirs. J Hospitality Tourism Management 2015:2219-2229.

12. Pensado-Leglise M. Cambios en los regímenes alimentarios en América Latina y riesgos para la seguridad alimentaria. LEISA, Rev Agroec 2011;27(3):30-33.

13. Josling T. The war on terroir: Geographical indications as a transatlantic trade conflict. Paper AES Annual Meeting; Paris. 2006.

14. Caputo V, Nayga RM, Scarpa R. Food miles or carbon emissions? Exploring labelling preference for food transport footprint with a stated choice study. Rev Aust J Agr Res Econ 2013;57(4):465-482. http://onlinelibrary.wiley.com/doi/10.1111/14678489.12014/pdf. Accesed Jun 14, 2015.

15. Pearson D, Henryks J, Trott A, Jones P, Parker G, Dumaresq, Dyball D. Local food: Understanding consumer motivations in innovative retail formats. $\mathrm{Br}$ Food $\mathrm{J}$ 2011;113(7):886-899. http://dx.doi.org/10.1108/00070701111148414 . Accesed Jun $15,2015$.

16. Barham B, Callenes M, Gitter S, Lewis J, Weber J. Fair trade/organic coffee, rural livelihoods, and the agrarian question: Southern Mexican coffee families. Transition World Develop 2011;39(1):134-145. 
17. Sacchi G, Caputo V, Nayga RM. Alternative labeling programs and purchasing behavior toward organic foods: The case of the participatory guarantee systems in Brazil. Sustainab 2015;(7):7397-7416. doi:10.3390/su7067397.

18. Feldmann C, Hamm U. Consumers' perceptions and preferences for local food: A review. Food Qual Pref 2015;(40):152-164. www.elsevier.com/ locate/foodqual. http://dx.doi.org/10.1016/j.foodqual.2014.09.014 . Accesed May 3, 2016.

19. Giraudel C, Caplat G. L'Agriculture Biologique et la Qualite. Approche juridique et normative. DRAEI/ 93031 Rapport final. Fac Droit et Scien Econ Limoges, Juin 1995.

20. Goodman M. Reading fair trade: political ecological imaginary and the moral economy of fair trade foods. Rev Polit Geogr 2004;(23)891-915.

21. Babcock B, Clemens R. Geographical Indications and property rights: Protecting valueadded agricultural products. MATRIC Briefing Paper 04-MBP 7 May 2004.

22. Sanz-Cañada J, Muchnik J. Geographies of origin and proximity: approaches to local agro-food systems. Cult Hist J 2016;5(1):1-19. e002 eISSN 2253-797X doi: http://dx.doi.org/10.3989/chdj.2016.002

23. Rodríguez J, Seain C. El debate Flichman-Braun: revisión y rescate a la luz del proceso de sojización." IV Jornadas Interdisciplinarias de Estudios Agrario y Agroindustriales. Buenos Aires: Ed electrónica FCEUBA; 2005.

24. Klitmovsky E. La teoría de la renta absoluta. Rev Econ Teo Prác. México 1984;(3):27140 http://www.izt.uam.mx/economiatyp/numeros/numeros/primera_epoca/03/ Consultado Jun 16, 2015.

25. MAGRAMA. Caracterización de la producción de alimentos diferenciados protegidos bajo denominaciones de origen y/o indicaciones geográficas protegidas. Ministerio de Agricultura, Alimentación y Medio Ambiente, NIPO: 280-15-060-4, Madrid, 2014.

26. SIGMA DOS. Estudio de opinión sobre la imagen y los hábitos de consumo de la carne de vacuno, principales resultados, Consultoría de SIGMA DOS, Análisis e Investigación S.L. para la Organización Interprofesional de la Carne de Vacuno, Madrid. 2017.

27. Atance I, Bardajíl I, Tió C. Instrumentos de diferenciación de la producción en el sector de la carne de vacuno. Rev Distrib Cons; 2002;(79):68-81.

28. Carne de la Sierra de Guadarrama, Sitio oficial: http://www.carneguadarrama.com/

29. Vías J, Sánchez J. Sierra de Guadarrama, viejos oficios para la memoria. Madrid: La Librería; 2016. 
30. Rojas CI. Hermenéutica para las técnicas cualitativas de investigación en ciencias sociales: una propuesta. Rev Espac Púb 2011;14 (31):176-189. http://www.redalyc.org/ articulo.oa?id=67621192010 Accesed Jun 16, 2015.

31. Calder BJ. Focus groups and the nature of qualitative marketing research. Jour Mark Res 1977;(14):353-64.

32. Sepúlveda W, Maza M, Pardos L. Factors that affect and motivate the purchase of quality-labelled beef in Spain. Meat Sci 2008;(80):1282-1289.

33. Mesías F, Escribano M, Gaspar P, Pulido F. Actitudes de los consumidores Extremeños hacia las carnes ecológicas, con IGP y convencionales. Arch Zootec 2008;57(218):139146.

34. Gracia A, Pérez L, SanJuán AI, Barreiro J. Análisis hedónico de los precios de la tierra en la provincia de Zaragoza. Rev Esp Est Agrosoc Pesq 2004;(202):51-69.

35. Ulloa R, Gil JM. Valor de mercado y disposición a pagar por la marca Ternasco de Aragón. Rev Esp Est Agrosoc Pesq 2008;(219):39-70.

36. Marette S, Crespi J, Schiavina A. The role of common labelling in a context of asymetric information, European Rev Agric Econom 1999;26(2):167-178.

37. Barjolle D, Sylvander B. PDO and PGI products: market, supply chains and institutions. Final Report European Commission, Brussels 2000; FAIR 1-CT95-0306. http://www.origin-food.org/pdf/pdo-pgi.pdf Accesed Jun 14, 2015.

38. Belletti G. Origin labelled products, reputation and heterogeneity of firms. In: Sylvander B, et al. editors. The socio-economics of origin labelled products: Spatial, institutional and co-ordination aspects. Proc 67th EAAE Seminar, October 28-30, Le Mans, France 1999:240-259.

39. Sandler T, Tshirhart J. The economic theory of clubs: An evaluative survey. J Econ Lit 1980;18(4):1481-1521 http://links.jstor.org/sci?sici=0022-0515\%28198012\%3A4\%3C 1481\%3ATETOCA\%3E2.0.CO\%3B2-Y. Accesed Jun 14, 2015.

40. Linck T, Barragán E, Casabianca F. De la propiedad intelectual a la calificación de los territorios: Lo que cuentan los quesos tradicionales. Rev Agroalimentaria; Venezuela 2006;2(22):99-109.

41. Belleti G, Marescotti A, Touzard JM. Geographical indications, public goods and sustainable development: The Roles of actors' strategies and public policies. World Develop 2017. 
42. Belletti G, Marescotti A, Paus M, Reviron S, Deppeler A, Stamm H, Thévenod-Mottet E. The effects of protecting geographical indications ways and means of their evaluation. Swiss Federal Institute of Intellectual Property, Berna, 2011. https://www.ige.ch/ fileadmin/user_upload/Juristische_Infos/e/publication_no_7_2nd_ed_Effects-ofProtecting-Geographical-Indications.pdf. Accesed Jun 16, 2015.

43. Sanz-Cañada J, Macías-Vázquez A. Quality certification, institutions and innovation in local agro-food systems: Protected designations of origin of olive oil in Spain. J Rural Studies 2005;(21):475-486. 